INT. J. PROD. RES., 2000, VOL. 38, NO. 5, 1071-1082

\title{
A wire-EDM maintenance and fault-diagnosis expert system integrated with an artificial neural network
}

\author{
J. T. HUANG† and Y. S. LIAO†*
}

In wire electrical discharge machining (Wire-EDM), some faults such as wirebreaking and unsatisfactory accuracy may still occur due to improper operations or inappropriate machine maintenance. A maintenance-schedule and fault-diagnosis system that integrates an artificial neural network (ANN) and an expert system (ES) is developed. It is time-saving in knowledge acquisition, is easy to maintain and is capable of self-learning. The occasions which call for machine maintenance are advised automatically. Suggestions to eliminate faults are proposed sequentially according to the inferred priority once a fault is taking place. Moreover, it can provide explanations.

\section{Introduction}

Although modern Wire-EDM machines have a high degree of automation and are quite robust, some faults may still occur due to improper operations by the users, and wear and damage of machine parts. The faults may include abnormal machining speed, deterioration of machining process (retreat of wire and wire breaking), unsatisfactory accuracy and surface roughness of machined parts, and even machine break-downs, etc. Even for a well-skilled operator, not to mention a novice, some faults of commercial Wire-EDM machines cannot be rejected instantly, because there are less straightforward and simple knowledge related faults and causes that can be learned easily. These faults will result in time delay, damage to workpieces and an increase of operating cost.

Applications of artificial intelligent (AI) have made significant progress in the past decade. An object-oriented ES, different from the record-oriented one, has many appealing features such as object-based modular structure, data abstraction, class, inheritance and instance of class, etc. Expert systems can integrate the expertise into an organized software and a neural network can handle difficult situations such as incomplete data sets or gray areas which the expert may not thoroughly understand. Neural networks are based on values and algorithms while expert systems are based on symbolic and heuristic reasoning.

Some conventional expert systems for fault diagnosis (Rumelhart et al. 1986, Angeli and Chatzinikolaou 1995) have been emphasized in the area of engineering. The importance of fault-diagnosis and operator assistance in an IF-THEN rulebased knowledge system for Wire-EDM (Snoeys et al. 1988) was also indicated.

Revision received June 1999.

$\dagger$ Department of Automatic Engineering, Fushin Institute of Technology, Toucheng, I-Lan, 261, Taiwan.

†Department of Mechanical Engineering, National Taiwan University, 1, Sec. 4, Roosevelt Rd. Taipei, 106, Taiwan.

*To whom correspondence should be addressed. e-mail: liaoys@ccms.ntu.edu.tw 
Although rule-based systems are certainly the biggest success story in AI, they still have drawbacks (Gaudill 1990), e.g. long development time, lack of fuzzy inference capability, etc. The application of artificial neural networks as the front end of expert systems for classification problems to overcome the bottleneck of knowledge acquisition (Gallent 1988) received considerable attention in 1988. The two paradigms of expert system and neural network have been combined into a hybrid expert system recently, and proven to be suitable for fault-diagnosis problems (Yoon et al. 1990, Yeh et al. 1993). Yeh et al. (1993) showed that an artificial neural network (ANN) can work satisfactorily as a knowledge-acquisition tool for diagnosis problems.

Wire-EDM is a complicated manufacturing process and many causes could lead to the occurrence of faults. Integration of an ANN algorithm and expert system will cut time in constructing the diagnosis system. It is capable of self-learning and providing an explanation function. Hence, the objective of this paper is to propose the architecture of a hybrid object-oriented expert system integrated with an artificial neural network algorithm in Wire-EDM. In the proposed system, the occasions which call for machine maintenance (e.g. replacement of ion exchange resin and diamond guides, etc.) are advised automatically so that faults can be prevented in advance. Suggestions to eliminate faults are proposed sequentially according to the inferred priority once a fault takes place. Moreover, it can also be used as a tool to educate novices and less experienced operators of Wire-EDM machines.

The paper is organized as follows. The algorithm of neural network and objectoriented expert system architecture are introduced briefly. Architecture of the WireEDM fault-diagnosis hybrid system is described and the inferring algorithm for the maintenance schedule and fault-diagnosis module will be discussed. Finally, two examples are given to show the applicability and validation of this method. In the future, the system developed will be included as modules of an intelligent Wire-EDM system under development.

\section{Method}

An expert system (Jackson 1990) is a computer program that represents and reasons with knowledge of some specialist subject with a view to solving problems or giving advice. Expert systems have advantages of using scarce expertise, cost reduction, improved quality, consistency of output, reduced downtime and increase output flexibility. An expert system consists of a user interface, inference mechanism, knowledge base, working memory, knowledge acquisition and an explanation facility, as shown in figure 1. Knowledge acquisition is probably the most difficult step and is time-consuming because of the necessity of eliciting a complete understanding of the decision-making process. An object-oriented expert system is easy to expand and maintain because of its reusability and inheritance ability. The internal structure of an object consists of two basic components: attribute and operation. Hence, objects can describe structural and behavioural knowledge simultaneously.

Neural networks explore many competing hypotheses simultaneously using massively parallel nets composed of many non-linear computational elements connected by links with variable weights, as shown in figure 2 (Lau 1992). A neural network typically consists of many simple processing elements, called neurons, that interact using weighted connections. A back-propagation learning algorithm, which is currently the most widely used algorithm for connectionist learning, is used in this research. 


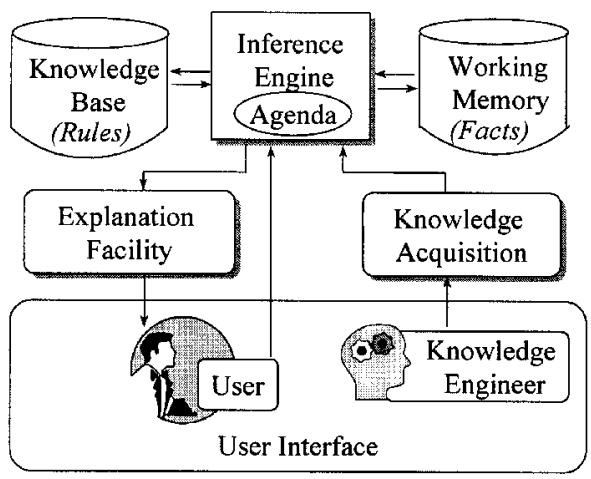

Figure 1. Structure of an expert system.

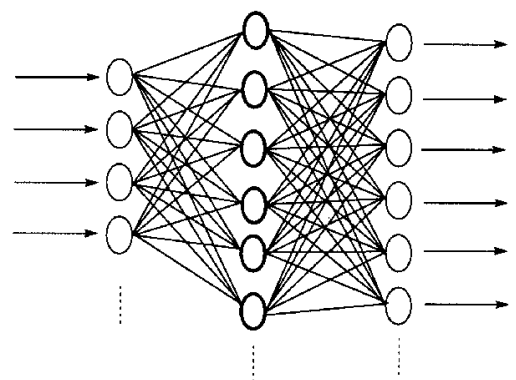

$\underline{\text { Input layer Hidden layer } \text { Output layer }}$

Figure 2. Configuration of neural network.

The hybrid expert system which incorporates the connectionist (ANN) and the logic programming paradigms (ES) has several advantages such as multi-inputs and non-linear programming, approximate reasoning capability, time-saving in construction, learning capability and an explanation capability.

\section{WMFES system}

The architecture of the Wire-EDM Maintenance \& Fault-diagnosis Expert System (WMFES) developed is shown in figure 3. There are two independent modules in this expert system: a maintenance schedule module and a fault-diagnosis module. The former employs the IF-THEN rule-based inference with objectoriented data structure and explicit knowledge base because inferring from the knowledge base is comparatively simple. The latter module was built with ANNbased learning and inference mechanism since the cause-effect relations of faultdiagnosis are more complicated. By using the ANN algorithm the efforts required to build the knowledge base can be greatly reduced. The learned knowledge is stored and appears as the 'weights' between features, hidden layer and causes. After inferring, the explanation is provided, which is built by the object-oriented IF-THEN rule-based algorithm, the same as maintenance schedule module. Two commercially available software packages-Smart Element (Nexpert object system), PCNeuron and a high-level $\mathrm{C}$ language program were used to construct the whole WMFES system. 


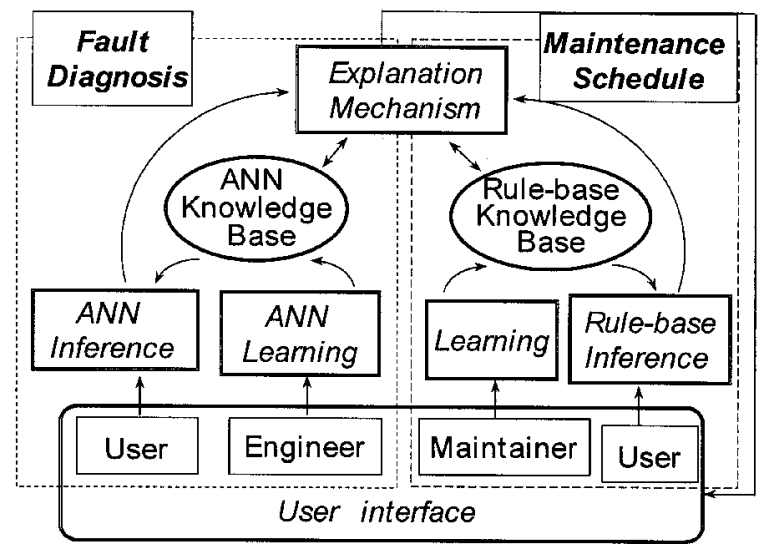

Figure 3. Architecture illustration of WMFES.

\subsection{Maintenance schedule module}

Proper maintenance of machine parts will decrease the faults-occurrence possibility for a Wire-EDM machine. Taking electricity-supply pieces as an example, it has to be checked every week or moved a distance after about 50 hours machining. Otherwise, the wear part will cause wire breaking or insufficiency of electricity supply. However, it is not easy and inefficient for operators to keep the service record. In this maintenance module, the occasions that call for machine maintenance (e.g. daily checking of the wire path, monthly checking and adjusting of the wire tension, etc.) are advised automatically. There are two kind of classes in this module: consumable machine parts and routine maintenance. The consumable machine parts are divided into three subclasses: water, structure and power system. Each class contains several objects that have the same properties as shown in figure 4 . The routine maintenance is divided into five subclasses namely daily, weekly, monthly, quarterly and annual maintenance as shown in figure 5. Table 1 indicates the meaning of properties of both classes.

This module will record machining time and remind operators to maintain or change some parts in advance. It also has the function of providing the stock

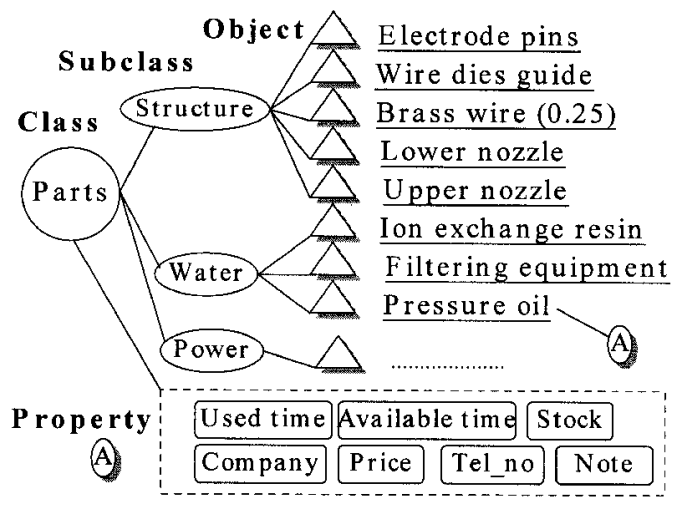

Figure 4. The relation of class and object of consumable parts of machine. 


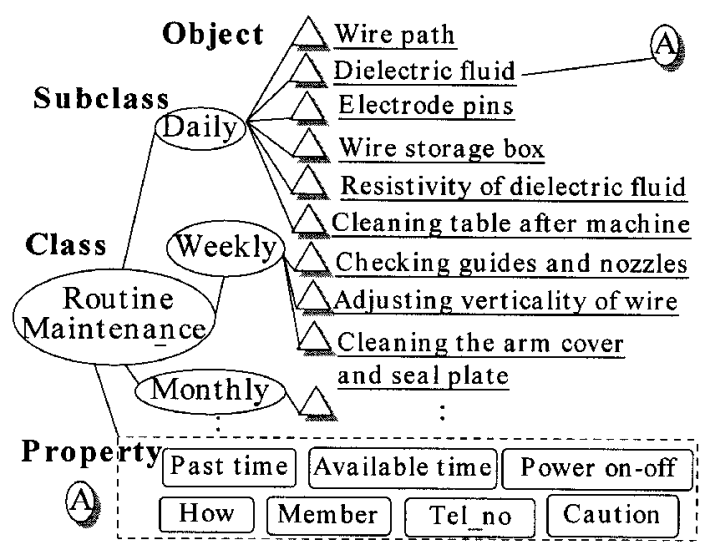

Figure 5. The relation of class, subclass, object and property of routine maintenance.

\begin{tabular}{|c|c|c|c|}
\hline \multicolumn{2}{|c|}{ Consumable part } & \multicolumn{2}{|c|}{ Routine maintenance } \\
\hline Property & Meaning & Property & Meaning \\
\hline Used time & Used time already & Past time & Past time already \\
\hline Available time & Life cycle of part & Available time & Maintenance cycle \\
\hline Stock & Number of new parts & Member & $\begin{array}{l}\text { Maintainer including operator, } \\
\text { engineer and service-person }\end{array}$ \\
\hline Company & Part-maker company & Power on-off & Power on or off while acting \\
\hline Tel_no & $\begin{array}{l}\text { Telephone number of } \\
\text { company }\end{array}$ & How & $\begin{array}{l}\text { Indicate maintenance } \\
\text { procedures }\end{array}$ \\
\hline Price & Price & Tel_no & Telephone number \\
\hline Note & Note & Caution & Caution \\
\hline
\end{tabular}

Table 1. Meanings of properties of two classes.

information and company of parts' suppliers. It will suggest to operators to buy new consumable parts at suitable times. According to different maintenance regimes, different maintainers including operator, engineer and service-person are needed. The routine maintenance will also instruct operators with the appropriate procedures to conduct the maintenance. Besides, this module also provides an interface to change the knowledge data such as available time according to an operator's experience. It also gives some explanation to the novice. Hence, this module can keep the machine in best condition so that high precision workpieces can be manufactured effectively. The algorithm of the maintenance schedule module is shown in figure 6. A practical example is provided in $\$ 3.3$.

\subsection{Fault-diagnosis module}

The Wire-EDM machine is a complicated machine that includes mechanical structure, power system and fluid system. Commercial Wire-EDM machines can provide alarm message and recommend the specific procedures to remove faults such as wrong NC program, low air pressure, etc. However, the cause of some alarm messages or faults cannot be found directly. Taking wire breakage as an example, many factors are needed to be checked step by step before rejecting them. Although some machines have the function of AWF (automatic wire feed 


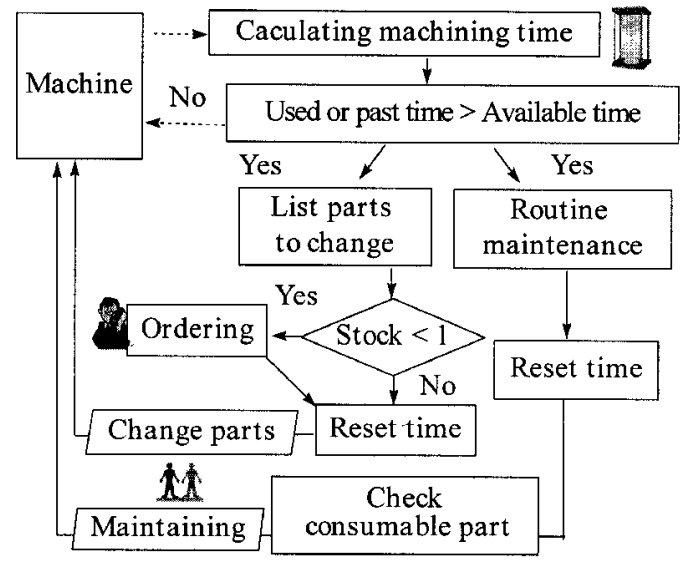

Figure 6. The inferring flow chart of maintenance schedule module.

mechanism), wire breakage will still happen again and again if the cause is not found and removed. Based on the ANN algorithm, this module allows operators to input observed partial and unknown data (defined as features here). Through the neural inference mechanism, possible causes will be advised subsequently according to their inferred weights. In this research, the faults include wire breaking, abnormal low machining speed, unsatisfactory surface, unsatisfactory accuracy and corner delay, etc. In the fault-diagnosis module developed, there are three mechanisms including building and learning, ANN-based inference and explanation mechanisms.

\subsubsection{Building and learning mechanism}

WMFES provides a learning interface for constructing the knowledge base at the very beginning to accumulate the knowledge of experienced Wire-EDM operators, handbooks (Fanuc 1996) and experimental results. Moreover, engineers can input new fault examples, which will change the weights of the inference mechanism between the layers. Reliability of the diagnosis results will be improved enormously when numbers of examples are increased. In this study, the method proposed by Yeh et al. (1993) was applied for constructing the learning and inference mechanism.

Data specification perhaps is the most important decision that any analyst makes in approaching a model. Taking wire-breaking as an example, eight causes have been identified as output data and 15 features as input data of wire breaking in this paper. One or more features can help the system find the most probable cause. Features have the characteristics of easy observation and examination. The features and causes of wire breaking are indicated in table 2 .

A single hidden layer is used and the number of hidden units could be taken as the same number of the input units (15 in this case) for wire-breaking. Learning and training examples are shown in table 3 , and a total of 50 examples were used. They were supplied to build and implement the knowledge base. After the learning process, a matrix-weight knowledge base was established. Figure 7 shows the converging trend of training and testing examples. The errors are between 0.1 and 0.2 . With more examples in future, the correctness of wire-breaking fault-diagnosis can be improved. Similarly, the knowledge base of the other faults can be established as well. 


\begin{tabular}{rlll}
\hline No. & \multicolumn{1}{c}{ Feature } & No. & \multicolumn{1}{c}{ Cause } \\
\hline 1 & In a minute after starting machining & a & Wrong machining parameters setting \\
2 & A few time after starting machining & b & Oxidized wire or workpiece \\
3 & Breaking position: electrode pin & c & Abnormal dielectric resistivity \\
4 & Breaking position: workpiece & d & Worn wire guide or chipped nozzle \\
5 & Breaking position: between lower guide & e & Wrong gap between the nozzle and \\
& and roller & & workpiece \\
6 & 'Bali-bali' sound before breaking & f & Worn electrode pin \\
7 & Wire sticks to the workpiece & g & Relaxation of wire mechanism \\
8 & Not move electrode pin for a long time & h & Workpiece falls and touches the rest of \\
9 & Long time no machining workpiece & & workpiece \\
10 & Multi-machining & & \\
11 & Not using the machining parameters table & & \\
12 & Dirty water in the clean-water tank & & \\
13 & Short alarm before breaking & & \\
14 & Workpiece with different height & & \\
15 & Unstable electrical discharge frequency & &
\end{tabular}

Table 2. Features and causes of wire breaking.

\begin{tabular}{|c|c|c|c|c|c|c|c|c|c|c|c|c|c|c|c|c|c|c|c|c|c|c|}
\hline \multicolumn{15}{|c|}{ Feature } & \multicolumn{8}{|c|}{ Cause } \\
\hline 1 & 2 & 3 & 4 & 5 & 6 & 7 & 8 & 9 & 10 & 11 & 12 & 13 & 14 & 15 & A & B & $\mathrm{C}$ & $\mathrm{D}$ & $\mathrm{E}$ & $\mathrm{F}$ & $\mathrm{G}$ & $\mathrm{H}$ \\
\hline-1 & +1 & +1 & -1 & -1 & -1 & -1 & -1 & -1 & -1 & +1 & -1 & +1 & -1 & -1 & 1 & 0 & 0 & 0 & 0 & 0 & 0 & 0 \\
\hline-1 & +1 & +1 & -1 & -1 & -1 & -1 & +1 & -1 & -1 & -1 & -1 & -1 & -1 & -1 & 0 & 0 & 0 & 0 & 0 & 1 & 0 & 0 \\
\hline+1 & -1 & -1 & +1 & -1 & +1 & +1 & +1 & +1 & -1 & -1 & -1 & +1 & -1 & +1 & 0 & 1 & 0 & 0 & 0 & 0 & 0 & 0 \\
\hline & $\vdots$ & & $\vdots$ & & $\vdots$ & & $\vdots$ & & & $\vdots$ & & & $\vdots$ & & & & $\vdots$ & & $\vdots$ & & $\vdots$ & \\
\hline
\end{tabular}

' +1 ' represents this feature is true, ' -' represents this feature is false.

Table 3. Input data (features) and results (causes) of wire breaking.

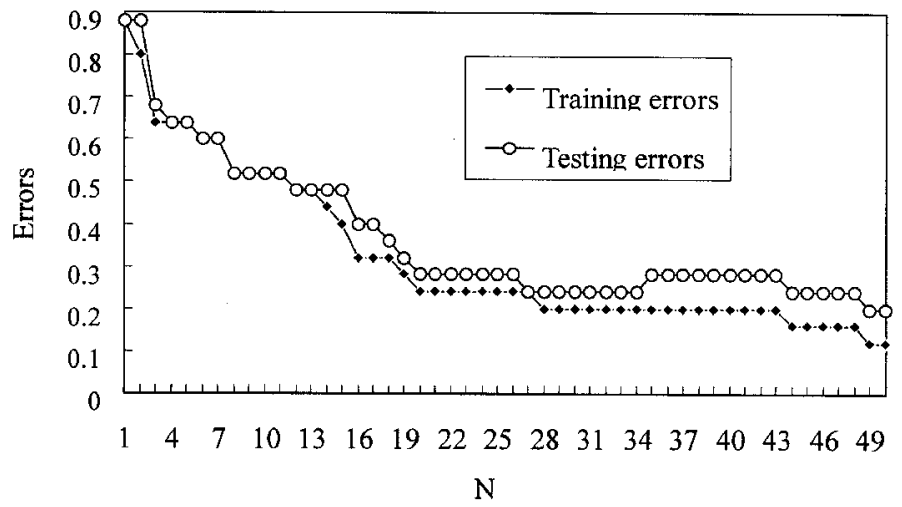

Figure 7. The converging trend of training and testing examples for wire-breaking.

\subsubsection{ANN-based inference mechanism}

The system is ready to diagnose new cases of fault after the network is trained with the back-propagation learning algorithm. A hybrid reasoning strategy that combines forward and backward reasoning schemes is used to build the inference mechanism. The algorithm is shown in figure 8 and described as follows.

(1) Input features. The user interface will list all the features on the screen and wait for the users to key in the value between -1 and +1 , where $-1,0$ and +1 represent false, unknown, and true, respectively. 


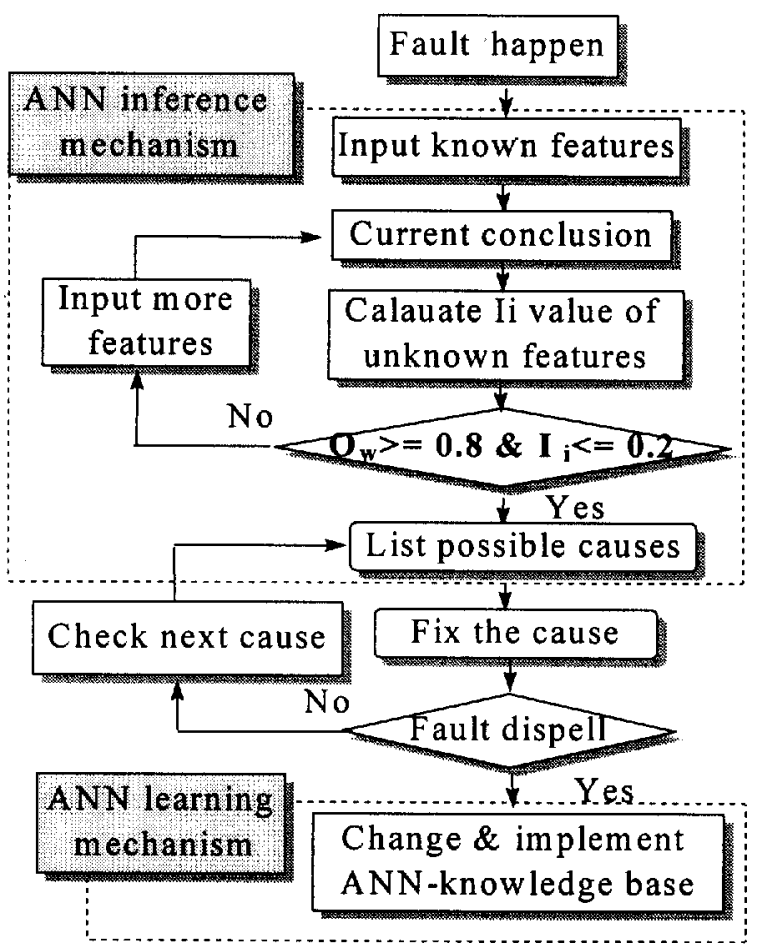

Figure 8. Flow chart of the inferring algorithm of fault-diagnosis module.

(2) Infer current conclusion. Based on the input data and the weights of the net, the output values will be inferred and displayed.

(3) Calculate the influence values. For each unknown input feature, the system will calculate its influence value on the maximum output value of all the output values. The influence value $(I)$ is defined as $I=\left|V_{i=1}-V_{i=-1}\right|$, where $V_{i=1}$ is the maximum output value when the input value of feature is +1 , and $V_{i=-1}$ is the maximum output value for the input value of feature -1 .

(4) Check the termination criterion. A conclusion is reached once the maximum output value is greater than the predetermined limit $\left(O_{w}\right)$ or the influence values is lower than the predetermined limit $\left(I_{i}\right)$; or users determine to terminate it because no further information is given. Otherwise, go to the next step.

(5) Input further information: According to the influence values of unknown features, the unknown input will be displayed for users to select the value in $(+1,-1)$. Then, go to step 2 .

\subsubsection{Explanation mechanism}

According to the ANN-inference result, the cause of the fault will be advised and the procedures to remove the fault will be suggested. At the same time, explanation is obtained according to the weights between features, hidden layer and causes. The explanation is constructed with the object-oriented rule based algorithm. The relation of class, object and properties of this mechanism is depicted in figure 9. Each cause of faults has the properties: action, member and explanation. The property provides operators the proper explanation. 


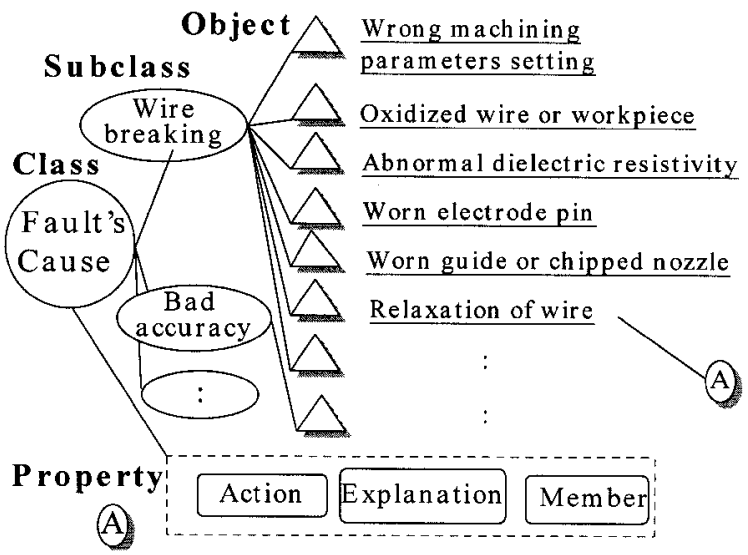

Figure 9. The relation of class, object and properties of the causes of faults.

\subsection{Examples}

For illustration purposes, two examples are given.

\subsubsection{Maintenance schedule}

The system will record the machining time and judge if the used_time (for consumable parts) or past_time (for maintenance requirements) is greater than the available_time or not.

\subsubsection{Check consumable part}

The following is the data of consumable parts before machining.

\begin{tabular}{llrrrrrrr} 
Name & Used_time & Available_time & Stock & Price & Tel_no| & Company| & Note \\
\hline brass_wire25 & $\mid$ & $25 \mid$ & $35 \mid$ & $4 \mid$ & $1000 \mid$ & $22779922 \mid$ & DAILEE & \\
electrode_pin & | & 5 & $50 \mid$ & $5 \mid$ & $3000 \mid$ & $22779922 \mid$ & DAILEE $\mid$ & $\mid$ \\
& $:$ & $:$ & $:$ & $:$ & $:$ & $:$ & $:$ \\
\hline
\end{tabular}

The following is the data of consumable parts after 10-hour machining.

Name | Used_time| Available_time| Stock| Pricel Tel_nol Companyl Note|

\begin{tabular}{|c|c|c|c|c|c|c|}
\hline brass_wire 25 & 0 & 35 & $4 \mid$ & 1000 & $22779922 \mid$ & DAILEE| \\
\hline electrode_pin & $12 \mid$ & 50 & 5 & 3000 & $22779922 \mid$ & DAILEE| \\
\hline & : & : & : & : & : & : \\
\hline
\end{tabular}

Then, the system reminds the operator to change the consumable parts.

Name l Notel Stock|

brass_wire25 | PLEASE CHANGE THIS PART NOW| 4|

After changing this consumable part, the stock number becomes 3 . When the stock is less or equal to 2 , which is determined by the programmer, then the system will show the following:

\begin{tabular}{lrrrrrr} 
Name & $\mid$ & Warning & Price & Companyl & Tel_no & Stock $\mid$ \\
\hline brass_wire25 & $\mid$ & BUY SOME PARTS IMMEDIATELY!| & $1000 \mid$ & DAILEE & $22779922 \mid$ & $2 \mid$ \\
\hline
\end{tabular}




\subsubsection{Check maintenance requirements}

The following is the data of maintenance schedule before machining

\begin{tabular}{|c|c|c|c|c|c|c|c|}
\hline Name & | Past_time| & ime| & Tel_no| & How $\mid$ & \multicolumn{2}{|c|}{ Member| Power_on_off| } & Caution| \\
\hline check_electrod_pin & $3 \mid$ & 50 & $23660443 \mid$ & HOW| & OPER ATOR | & Off & | \\
\hline adjust_wire_verticality & $61 \mid$ & 70 & $23660443 \mid$ & HOW| & ENGINEER | & $\mathrm{ON}$ & r \\
\hline & : & : & : &. & : & : & : \\
\hline
\end{tabular}

The following is the data of maintenance schedule after 10 hour machining Name | Past_time| Available_time| Tel_nol How $\mid$ Member| Power_on_off| Caution|

\begin{tabular}{rrrrrrrr}
\hline check_electrod_pin & $13 \mid$ & $50 \mid$ & $23660443 \mid$ & HOW $\mid$ OPERATOR & Off| & | \\
adjust_wire_verticality & $0 \mid$ & $70 \mid$ & $23660443 \mid$ & HOW $\mid$ ENGINEER & ON $\mid$ & | \\
& $:$ & $:$ & $:$ & $:$ & $:$ & $:$ & $:$ \\
\hline
\end{tabular}

The system will remind operators to conduct the routine maintenance.

Name

Caution| Member| Power_on_off|

How

adjust_wire_verticality | DO MAINTENANCE NOW| ENGINEER |

$\mathrm{ON} \mid$ Using verticality instrument|

\subsubsection{Fault Diagnosis}

An example of wire-breaking fault-diagnosis is given in the following to demonstrate the consulting procedures. All the data input by users are underlined and the inferring data are bracketed.

-Please input maximum output limit $\left(O_{w}\right)=\underline{0.80}$

-Please input maximum influence limit $\left(I_{i}\right)=\underline{0.20}$

-Please input initial features $(+1$ for true, 0 for unknown and -1 for false $)-$

1. In a minute after starting machining:

2. Some time after starting machining:

3. Breaking position: electrode pin:

4. Breaking position: workpiece:

5. Breaking position: between lower guide and roller:

6. 'Bali-bali' sound before breaking:

7. Wire sticks to the workpiece:

8. Not move electricity pin for a long time:

9. Long time no machining:

10. Multi-machining:

11. Not using the machining parameters tables:

12. Dirty water in the clean-water tank:

13. Short alarm before breaking:

14. Workpiece with different height:

$\frac{1.000}{-1.000}$
$\frac{-1.000}{1.000}$
$\frac{-1.000}{\underline{1.000}}$
$\underline{0.000}$
$\underline{\underline{0.000}}$
$\underline{0.000}$
$\frac{-1.000}{\underline{0.000}}$
$\underline{\underline{0.000}}$
$\underline{0.000}$
$\underline{0.000}$
$\underline{0.000}$

15. Unstable electrical discharge frequency:

The possible results are

2. Oxidized wire or workpiece:

1. Wrong machining parameters setting:

3. Abnormal dielectric resistivity

4. Worn wire guide or chipped nozzle

5. Wrong gap between the nozzle and workpiece:

8. Workpiece falls and touches the rest of workpiece 
7. Relaxation of wire mechanism:

6. Worn electrode pin:

The maximum output $\left(O_{w}\right)$

The maximum influence $\left(I_{i}\right)$

$(0.560925)$

Please input further data $(*$ represents influence value $(I))$

9. Long time no machining $(* 0.561)$ :

$\underline{1.000}$

11. Not using the machining parameters tables $(* 0.305)$ :

$\underline{0.000}$

7. Wire sticks to the workpiece $\left({ }^{*} 0.292\right)$ :

$\underline{1.000}$

14. Workpiece with different height $(* 0.256)$ :

8. Not move electricity pin for a long time $(* 0.246)$ :

13. Short alarm before breaking $(* 0.161)$ :

15. Unstable electrical discharge frequency $(* 0.132)$ :

$\underline{0.000}$

12. Dirty water in the clean-water tank $\left({ }^{*} 0.086\right)$ :

$\underline{0.000}$

$\underline{0.000}$

$\underline{0.000}$

$\underline{0.000}$

The possible results are

2. Oxidized wire or workpiece:

$(0.814)$

1. Wrong machining parameters setting:

$(0.186)$

3. Abnormal dielectric resistivity:

$(0.109)$

4. Worn wire guide or chipped nozzle:

$(0.108)$

7. Relaxation of wire mechanism:

8. Workpiece falls and touches the rest of workpiece:

5. Wrong gap between the nozzle and workpiece:

6. Worn electrode pin:

The maximum output $\left(O_{w}\right)$ :

The maximum influence $\left(I_{i}\right)$ :

$(0.193848)$

\section{Explanation mechanism}

IF

9. Long time no machining $=$

4. Breaking position: workpiece $=$

7. Wire sticks to the workpiece $=$

1. In a minute after starting machining $=$

1.000

\section{THEN}

The most possible cause of wire breaking is oxidized wire or workpiece $(0.814)$

Name|

oxidized_wire_or_workpiece | Please change the wire or finish the workpiece's surface
Because wire breaking takes place at the beginning of machining and wire sticks to workpiece surface with a 'Bali-bali' sound. This is probably due to the concentrating electrical discharge on the non-plane surface of wire or workpiece, which had oxides or black iron on their surfaces.

\section{Conclusions}

This paper proposed a prototype ANN-based expert system for the maintenance schedule and fault-diagnosis of Wire-EDM. Owing to the advantages of ANN algorithm and expert system, this system is a powerful development tool for a complicated consulting system such as a Wire-EDM process. 
The system developed will help operators, even for novices and trainees in WireEDM, to maintain machines well, and eliminate faults sooner. Hence, the operating time can be minimized and the cost can be lowered. In the future, more examples will be provided so that the inferring reliability and accuracy of the system can be improved.

\section{References}

ANGeli, C. and ChatZinikolaou, A., 1995, An expert system approach to fault diagnosis in hydraulic systems. Expert Systems, 12, 323-329.

Fanuc LTD, 1996, Fanuc Robocut a Series Maintenance Manual (Japan: Fanuc Ltd).

Gallent, S. I., 1988, Connectionist expert systems. Communications of the ACM, 31, 152169.

Gaudill, M ., 1990, Using neural nets: hybrid expert networks (Part 6). AI Expert, 49-54.

JACKSON, P., 1990, Introduction to Expert Systems, 2nd edn (Wokingham: Addison-Wesley), p. 3.

LAU, C., 1992, Neural Networks: Theoretical Foundations and Analysis (New York: IEEE Press).

R UMelhaRT, D . E., Hinton, G . E. and Williams, R . J ., 1986, Learning internal representation by error propagation, in D. E. Rumelhart and J. L. McLelland (eds), Parallel Distributed Process: explorations in the microstructure of cognition (Cambridge, MA.: MIT Press), pp. 318-362.

Snoeys, R., Dekeyser, W. and Tricarico, C., 1988, Knowledge-based system for Wire EDM. Annals of the CIRP, 37/1, 197-202.

Yen, Y.C., K U O, Y. H. and H su, D. S., 1993, Building KBES for diagnosing PC pile with artificial neural network. Journal of Computing in Civil Engineering, ASCE. 7, 71-93.

Yoon, Y., Brobst, R. W., Bergstresser , P. R . and Peterson, L. L., 1990, A connectionist expert systems for dermatology diagnosis. Expert Systems, 22-31. 